\title{
Dual-tasking postural control: Aging and the effects of cognitive demand in conjunction with focus of attention
}

\author{
Oliver Huxhold*, Shu-Chen Li, Florian Schmiedek, Ulman Lindenberger \\ Center for Lifespan Psychology, Max Planck Institute for Human Development, Berlin, Germany \\ Received 7 June 2005; received in revised form 3 January 2006; accepted 5 January 2006 \\ Available online 20 January 2006
}

\begin{abstract}
Postural control in everyday life is generally accompanied by posture-unrelated cognitive activity. Thus, mild forms of dual-tasking postural control are the norm rather than the exception. Based on this consideration and available evidence, we propose and empirically examined, in young and old adults, a non-monotonic, U-shaped relation between the efficacy of postural control and concurrent cognitive demands that reflect opposing trends of the effects of attention focus and attentional resource competition. When instructed to perform an easy cognitive task that presumably shifted the focus of attention away from posture control, the center of body pressure (COP) excursions decreased both in young and older adults relative to a single-task baseline where the focus of attention was explicitly directed towards the postural control task itself. However, when performing more demanding cognitive tasks, older adults showed increased COP displacements, in line with the predicted Ushape function, whereas young adults did not. We outline mechanisms linking postural control to cognitive demand and suggest routes for future investigation.
\end{abstract}

(C) 2006 Elsevier Inc. All rights reserved.

Keywords: Aging; Attention; Automaticity; Balance; Working memory; Body representation; Resource competition

\section{Introduction}

Postural control, a ubiquitous task that humans perform daily, involves complex processes that require dynamic integration of visual, proprioceptive, and vestibular sensory information (see [31,37], for review). A well-established view suggests that the brain utilizes internal representations of forward motor commands and multi-sensory feedbacks to monitor, update, calibrate, and maintain the body's position (e.g., [37,72,74]). Findings derived from spatial-neglect patients who suffer from postural instability suggest in particular the involvement of parietal cortical functions in postural control probably implicating internal body representations $[27,38,44,65,66,73,75]$. In addition to maintaining the internal body representation in $3 \mathrm{D}$ space, parietal lobe functions may also underlie covert preparatory

\footnotetext{
* Corresponding author. Lentzealle 94, D-14195 Berlin, Germany. Tel.: +49 3082406 241; fax: +49 308249939 .

E-mail addresses: hux@mpib-berlin.mpg.de (O. Huxhold), shuchen@mpib-berlin.mpg.de (S.-C. Li), schmiedek@mpib-berlin.mpg.de (F. Schmiedek), seklindenberger@mpib-berlin.mpg.de (U. Lindenberger).
}

motor attention [55,56], somewhat analogous to their roles in covert orienting of visual attention [46].

Keeping balance of the upright stance is a highly practiced daily task for healthy adults and is effectively performed without overt (e.g., conscious) attentional control in most circumstances. Moreover, postural control in daily situations routinely takes place while at least one other concurrent task is being performed: for instance, standing while thinking or standing while talking. Thus, "dual-tasking postural control" - the regulation of postural stability while performing some other concurrent task - is the rule rather than the exception for the postural control system. Nevertheless, empirical evidence shows that attention might be needed in postural control to some degree for sensory integration and selection between conflicting sensory information (e.g., $[50,59,64])$ or to compensate for perturbations to the postural control system (see [71], for review). Therefore, maintaining an upright stance may tax cognitive factors, such as attentional processes, when the standing conditions are challenging or when attentional interference between postural control and cognitive processes is high [71]. This is consistent with related cognitive and neurocognitive evidence on attentional limitations in 
performing multiple tasks at the same time that has been gathered in fields other than human movement science [42,45].

\subsection{Dual-task attentional sharing between postural control and cognition}

The attentional demand for regulating postural sway is typically examined with the dual-task paradigm, which presumes that cognitive functions and postural control (as well as other sensorimotor functions, such as walking) compete for limited attentional capacity (see [22,71], for reviews). Thus, when one needs to maintain the balance of upright stance while performing a concurrent cognitive task, attention is divided between the sensorimotor and cognitive tasks. The efficacy of postural control under the dual-task condition may decrease in comparison to single-task performance to varying degrees according to the processing demands of concurrent cognitive processing, as the sharing of attentional resources between two domains of functioning reduces the amount of attention that is available for postural control.

Given the seemingly convincing and straightforward notion of competition for attentional resources between postural control and cognition, it may come as a surprise that the empirical evidence is far from being unequivocal. Some research suggests that dual-tasking postural control compromises balance performance $[2,7,32,36,39,40,43,59,60,68]$, as the resource view would predict. For instance, in support of the attention resource competition view, Andersson et al. [2] found that performing a concurrent visuospatial search task decreased balance performance in middle-aged adults $\left(M_{\mathrm{age}}=40.4\right.$ years $)$ under a challenging condition with attenuated proprioceptive sensory inputs (i.e., standing on a sway-referenced platforms). However, other studies found that performing a concurrent cognitive task while standing actually enhanced postural control $[1,4,8-11,14,51,52,63,70,76]$. For example, examining the effects of a variety of working memory tasks including a visuospatial task, Dault et al. [8] found reduced center of body pressure $(\mathrm{COP})$ displacements in young adults $\left(M_{\mathrm{age}}=23.0\right)$ under dual-task situations compared to the single-task condition regardless of the difficulty of the postural control task.

Thus, taken together, the inconsistency in current empirical literature on dual-tasking postural control suggests that the relation between postural control and mental processing merits further scrutiny [51]. In this regard, a number of researchers have postulated that the relationship between postural control and cognitive demand in dual-task situations could be U-shaped (e.g., $[11,51,68,69])$. According to this view, postural control performance is either improved or attenuated depending on whether the cognitive demand of the secondary task is low or high, respectively. Examining this hypothesis across studies by systematic comparisons of published evidence is problematic because most of the relevant studies differ widely in critical design features. Also, factors that affect the relation between postural control and cognition in addition to cognitive demand, such as age or expertise, are rarely varied within the same study.

\subsection{Potential factors affecting resource sharing between postural control and cognition}

A review of the literature suggests that the interactions between mental processes and postural control can be modulated by factors such as stimulus and response modalities (e.g., $[10,61,62,80]$ ), difficulty of the concurrent secondary task (see [72], for review), postural constraints affecting the difficulty of the postural task (e.g., $[9,19,50,68,79]$, individual differences in sensorimotor expertise (e.g., [68]), and aging (e.g., $[11,14,22,25,28,32,36,63])$. A number of studies considered the influence of experimental procedural factors of the concurrent task (e.g., stimulus presentation and response modes) on posture control. It has been shown that oral responses to cognitive tasks performed while standing worsens postural control performance $[10,80]$. In contrast, the need to fixate a visually presented stimulus appears to improve postural control [61,62]. Differences in such procedural factors of the concurrent secondary task alone, however, cannot give a full account of the inconsistency between findings regarding the effect of cognitive demands on postural control. Even among studies that controlled for the influences of such procedural factors, some reported impaired (e.g., [7]), whereas others found improved (e.g., [51]) postural control performance while dual-tasking.

Given this seemingly paradoxical pattern of findings, the motivation for this study is to better understand how concurrent cognitive processing can affect postural control in both positive and negative directions. In order to directly test the notion of a U-shaped relation between postural control and cognitive demands as well as factors that may modulate this relation, we jointly considered adult age differences in attentional capacity, locus of attentional focus, and the extent of cognitive demand while holding stimulus presentation and response modes of the concurrent tasks as constant as possible.

\subsubsection{Adult age differences in attentional capacity}

It is conceivable that dual-tasking effects are modulated by individual differences in attentional capacity and associated neural substrates. If the cognitive demand of a given concurrent secondary task does not sufficiently tax the individual's attentional capacity, its effect on postural control is very unlikely to be observed. In other words, at a given level of cognitive demand, negative effects of the concurrent secondary cognitive task on postural control may only be observed in individuals whose attentional capacity is more limited. Aging reduces the efficacy of sensory and muscular systems involved in postural control and attenuates their harmonious interplay [26,71]. In addition to aging-related declines in peripheral sensory and muscular systems, aging-related declines in the parietal cortex, the cortical region underlying the internal body representation in space are evident at neurochemical $[13,16]$, neuroanatomical [67], and functional levels (e.g., [18]). It has been hypothesized that losses at these various levels lead to a higher need for cognitive involvement in sensorimotor processing among older adults compared to young adults (see [22], for review). At the same time, impaired cognitive capacities and the associated losses in prefrontal working memory and attention functions 
commonly found in older adults (e.g., $[15,49,57])$ may, in turn, impair the successful employment of attentional resources for effective postural control $[25,30]$. Such reciprocal influences between attenuated sensory integration, sensorimotor functions' greater reliance on cognitive processes, and more limited cognitive capacity in later adulthood probably contribute to increased couplings between cognitive and sensorimotor processes (e.g., $[3,20,24,25])$. In summary, parallel declines in various levels of brain mechanisms as well as cognitive and sensorimotor processes during aging has motivated researchers to investigate the relations between neurocognitive mechanisms underlying attention and postural control (see [21], for review). Thus, quasiexperimental manipulation of adult age differences in attentional capacity may help explaining why dual-task costs in postural control were found in some cases but not in others.

\subsubsection{Locus of attentional focus}

To understand dual-tasking benefits on postural control, we consider the role of the secondary task in directing the individual's overt attentional focus away from the highly practiced, automatized postural control process. It is a common practice in research on postural control that participants are instructed to stand as still as possible. Most likely, this instruction directs participants' attention internally towards the actual execution of relatively automatized postural control processes. A number of studies have consistently found that postural control performance was worse when participants' attention was directed to postural control performance in internal-focus conditions as opposed to external-focus conditions where attention was diverted to a supra-postural task $[34,35,53,77,78]$. A possible conclusion is that directing overt attention to a highly automatized process, such as postural control under regular standing conditions, actually reduces the efficacy of this process.

\subsubsection{Cognitive demands of the secondary task}

Despite their high degree of automaticity, postural control processes may still require motor preparatory attention to facilitate multi-sensory integration and the generation of motor commands (cf. [55,73]). Conceivably, when a secondary cognitive task sufficiently or overly strains the individual's attentional capacity, covert motor preparatory attention that is part of the automatized process of postural control may be affected as well. Thus, it is reasonable to expect that the extent of the secondary task's cognitive demand may, in turn, set limits on the beneficial effect of dual-tasking on postural control. Whereas an easy secondary task may beneficially increase the efficacy of postural control by providing an external attention focus, a more demanding secondary task may have a negative consequence on postural control, due to attentional resource competition between cognitive and sensorimotor processing.

Visual sensory information is an integral part of postural control. Therefore, some studies have argued that postural control is more easily disturbed by a secondary task that involves visual-spatial rather than verbal information $[17,32]$. For instance, there is evidence for higher COP displacements under the condition of processing a concurrent spatial task than in the condition of processing a verbal task [17,32]. Other studies have found, however, no differences between verbal and spatial dual-task conditions $[8,12,79]$ or less COP displacements in spatial than in verbal processing conditions $[14,60]$. Again, it is of particular importance to equate the general cognitive demands, or difficulty, of verbal and spatial tasks used in dual-task studies in order to compare the "pure" difference in the effects of concurrent verbal and spatial processing. The current empirical ambiguity regarding the spatial-verbal dissociation may, in part, be due to differences in task difficulty unrelated to their verbal or spatial nature.

\subsection{Key hypotheses}

Given evidence of aging-related decline in sensorimotor and cognitive function, we expect older adults will perform below the level of young adults in postural control and cognitive tasks. Confirmation of this hypothesis will serve as a manipulation check to show that average performance levels of the present samples of young and older adults are typical of their age groups. Of specific interest, however, is the interaction between the quasi-experimental, between-subjects factor of attentional capacity differences, indexed by the two age groups, and the experimental, within-subjects factor of dual-task performance.

Dual-task effects can manifest themselves either in postural control or cognitive performance. In order to obtain an unequivocal interpretation of age differences in the relation between dual-tasking and postural control, age differences in dual-task costs in the cognitive domain must be considered. Moreover, cognitive performance could either be measured in terms of accuracy or in terms of processing speed. Thus far, most research on dual-tasking postural control has been primarily limited to a single cognitive performance indicator: accuracy. In the current study, both indicators are considered jointly to obtain a more precise picture of cognitive performance. Given that the postural control task we examined was standing without further postural constraint, we expected no or only small age differences in dual-task effects in the cognitive domain, an outcome that would enhance the interpretability of age differences in the postural domain.

With regard to dual-task effects in the domain of postural control, we propose that the ambiguity in current empirical findings cannot be fully accounted for by procedural factors of the concurrent secondary task (e.g., stimuli or response modes). Instead, we posit a complex interaction between task characteristics and age. We expect that adult age differences in attentional capacity, the locus of attentional focus, and the demand characteristics of the secondary task all contribute to the complex relation between postural control and mental processing. Specifically, we hypothesize that performing a concurrent secondary task that is relatively low in cognitive demand may benefit postural control by directing individuals' overt attention away from postural control processes that are usually carried out automatically. However, further increments in the cognitive demands of the secondary task will eventually result in attentional resource competition, thereby hampering the regulation of postural sway. In other words, and in line with previous suggestions $[11,51,68,69]$, we predict a $\mathrm{U}$-shaped relation between the cognitive demands 
of the secondary task and postural control. We further assume that the extent of this relation may be modulated by individual differences in attentional capacity. Given older adults' more limited attentional capacity and their declining posture control processes, we expect that attentional competition between postural and cognitive tasks emerges at lower levels of cognitive task demands in older than in young adults. Hence, we expect that the rising part of the U-shaped function relating levels of cognitive demand to measures of postural sway will emerge at lower levels of cognitive task difficulty in older adults than in young adults.

\section{Material and methods}

\subsection{Participants}

Originally, 41 adults were sampled from the participant pool of the Max Planck Institute for Human Development, Berlin, Germany. The experiment was undertaken with written consent of each participant. None of the participants had previously taken part in studies of posture or gait, and all were naïve with regard to expected results of the study. The ethics committee of the Max Planck Institute for Human Development approved the study. Two participants were excluded from the analyses: an older man because of exceptionally high COP displacements (approximately 3.7 S.D. above the mean of the old adult age group) and an older woman because of lack of compliance to task instructions. The effective sample, thus, consisted of 19 older adults $\left(M_{\mathrm{age}}=69.80\right.$, S.D. $\cdot$ age $=3.39 ; 10$ males and 9 females $)$ and 20 young adults $\left(M_{\mathrm{age}}=24.52\right.$, S.D .age $=2.63 ; 10$ males and 10 females). The two age groups differed in terms of height $\left(M_{\text {height }}=1.66 \mathrm{~m}\right.$, S.D.height $=0.07$ for older adults and $M_{\text {height }}=1.76 \mathrm{~m}$, S.D.height $=0.09$ for young adults $)$ and weight $\left(M_{\text {weight }}=74.6 \mathrm{~kg}\right.$, S.D. weight $=13.7 \mathrm{~kg}$ for older adults and $M_{\text {weight }}=68.4 \mathrm{~kg}$, S.D. weight $=9.4 \mathrm{~kg}$ for young adults). The participants received $€ 15$ (approximately $\$ 15$ ) for their participations.

\subsection{Apparatus}

Postural control performance was measured by a Kistler force platform (Type 9286AA, Kistler Instrumenten AG, Winterhur, Switzerland; size $60 \mathrm{~cm} \times 40 \mathrm{~cm}$ ). Twelve sensors built into the force platform measured mediolateral, anterior-posterior, and vertical components of ground reaction forces and momentums. A measurement computer ( $\mu$-MUSYCS Inc.; m-M-S-Eth-RJ45) sampled their signals at a rate of $80 \mathrm{MHz}$ and calculated $x-y$ coordinates of center of body pressure (COP) positions for every millisecond. The area traveled by the COP reflects the efficacy of postural control, with larger COP displacements indicate worse postural control performance.

At each trial, fixation cross as well as visual stimuli were presented at the center of a 17 -in. computer screen at $50 \mathrm{~cm}$ distance. Participants responded to cognitive tasks with two hand-held button boxes. The measurement computer concurrently stored stimulus presentation times and the participants' buttonbox responses. The sampling rate for the reaction time data was $1000 \mathrm{~Hz}$. The cognitive tasks were tested in sitting and standing conditions.

\subsection{Study design and procedure}

Participants were tested individually within a single session that lasted about $1.5 \mathrm{~h}$. At first, instructions of the cognitive tasks were given and every participant did at least one practice block per task while seated. The experiment included three single-task cognitive conditions while seated and five experimental conditions in which the participants stood on the force platform. The single-task cognitive conditions consisted of a digit choice-reaction time task, a 2-back digit working memory task, and a 2-back spatial working memory task performed in seated position. Standing conditions included a single-task standing and focusing a fixation cross condition, a dual-task standing while watching digits condition, and another three dual-task conditions that comprised standing on the force platform in combination with each of the three cognitive tasks. Every condition was measured within a block of four trials lasting $68 \mathrm{~s}$ each. The trial duration used in the study appears to be somewhat longer than the duration typically used in other studies (however, see also $[8,10,29,40]$ ). This trial duration was chosen to allow for the concurrent assessment of cognitive performance within dual-task conditions (e.g., with the $n$-back working memory task). Furthermore, some studies have demonstrated that longer trial durations yield more reliable assessments of postural sway [5].

It was not possible to fully counterbalance all experimental conditions, given the large number of conditions involved. However, special care was taken to control for potential sequence effects in the set of between-condition comparisons of interest using a nested (e.g., incomplete) counterbalancing scheme. To rule out sequential effects arising from the order of single-task and dual-task conditions, half of the participants in each of the two age groups first performed single-task cognitive conditions followed by various standing conditions, whereas the other half began with the standing conditions. The sequence of the three single-task cognitive conditions was counterbalanced across subjects. Within the standing conditions, every condition was equally often preceded or followed by any other condition across participants. Furthermore, within dual-task conditions, verbaltask blocks and spatial-task block were counterbalanced.

\subsection{Cognitive and postural control tasks}

We chose three different verbal-based cognitive tasks that differed with regard to the levels of their cognitive demand. A visuo-spatial task was employed as well. Relevant task parameters (e.g., stimulus presentation and response modes) were kept constant across tasks. In all conditions, participants responded with two hand-held button-boxes. In every experimental trial, participants were shown a random series of 22 stimuli on the computer screen. The random series presented in a given experimental trial-by-task combination was identical for every participant. The presentation time for every stimulus was $500 \mathrm{~ms}$, the inter-stimulus-interval (ISI) was $2500 \mathrm{~ms}$ in all conditions. Therefore, the maximal duration for the participants to respond amounted to $3000 \mathrm{~ms}$ before the next stimulus appeared. Participants responded to a target by pressing the button held in their dominant hand. Pressing the button held in their non-dominant hand identified non-targets. Responses faster than $100 \mathrm{~ms}$ were treated as errors.

\subsubsection{Choice-reaction time task}

In the choice-reaction time task, participants were shown random series of 22 digits per trial ranging between one and nine. The digits one to three were the assigned targets. The responses to the very first two digits in a trial were excluded from the analyses in order to have the same number of responses in the choice-reaction time task and in the two 2-back working memory tasks.

\subsubsection{Digit 2-back working memory task}

In every trial of the digit 2-back working memory task, a series of 22 digits ranging between zero and nine was presented. The participants had to indicate a target if a presented digit was identical to the one shown two steps back in the sequence. By definition, the first two digits of a series could only be non-targets. Participants were instructed to respond to these stimuli but these responses were excluded from the analyses.

\subsubsection{Spatial 2-back working memory task}

In the spatial 2-back working memory task, a dot appeared in one of eight locations of a three-by-three grid (the middle location of the grid was not used). A series of 22 locations was presented and the participants had to indicate a target if a location of the dot was identical to the one shown two steps back in the sequence. For reasons mentioned above, responses to the first two locations presented in a trial were excluded from the analyses.

Postural control performance was measured in five different conditions. Special care was given with regard to the stance position in which COP displacements were assessed. Large interindividual differences in feet positions can be found if participants are completely free to choose the stance position in which they prefer to be measured $[6,33,41]$. Older adults have the tendency to place their feet closer together and to have a wider angle between their feet than young adults [33]. A narrower feet placement is associated with a poorer postural control performance in contrast to a wider distance between feet. However, differences in distances between the feet have to be relatively large to significantly affect COP displacements. The range of optimal inner feet distances is more than 
$10 \mathrm{~cm}$, for instance [41]. Moreover, thus far it is unclear whether the age difference in average stance position is not an indication of an adaptive adjustment of older adults to their diminished postural control capacity [33]. Therefore, in our study we tried to find a middle ground between equating stance positions for reasons of comparability and simulating a "natural" stance behavior for each individual. At the beginning of the postural control assessment, participants were instructed to place their feet apart at approximately shoulder-width length with their toes pointing away from their body center. Within these constraints every participants was asked to find his or her own preferred standing position. The participant's chosen stance position was marked and subsequently used throughout the experiment. Thus, we "normalized" the stance position across different experimental conditions within each participant, but allowed individuals to choose their preferred stance position using a common guideline. Participants fixated the middle of a 17 -in. computer screen at approximately $50 \mathrm{~cm}$ distance. Gaze was individually adjusted to eye level by adjusting the vertical distance between the computer screen and ground. In all conditions, participants held button boxes in both hands with arms hanging relaxed beside their body. In all conditions, participants were instructed to stand as still as possible. Furthermore, in dual-task conditions participants were told to give equal emphasis to cognitive and postural control performance.

\subsubsection{Standing only condition}

Participants stood on the force platform and saw a fixation cross in the middle of the computer screen. The fixation cross was blue imbedded in a grey background and had a size of $12 \mathrm{~cm} \times 17 \mathrm{~cm}$. The participants were instructed to fixate at the center of the fixation cross. The center's position was identical to the location where the visual stimuli occurred in the dual-task conditions to make sure that differences in postural control between single and dual-task conditions were not driven by stabilizing effects of looking $[61,62]$.

\subsubsection{Standing and digits condition}

Participants stood on the force platform and watched a random series of digits ranging from one to nine presented on the computer screen. They were instructed to stand as still as possible while simply watching the digits.

\subsubsection{Standing and digit choice RT condition}

Participants stood on the force platform and concurrently performed the choice-reaction time task as fast and as accurately as possible.

\subsubsection{Standing and digit 2-back working memory condition}

Participants stood on the force platform and concurrently performed the digit 2-back working memory task.

\subsubsection{Standing and spatial 2-back working memory condition}

Participants stood on the force platform and concurrently performed the spatial 2-back working memory task.

\subsection{Data analysis}

In the cognitive domain, the three main dependent variables were response accuracy (percentage correct), reaction time (ms), and a unit-weighted combined score of accuracy and reaction time scaled in a T-metric (i.e., a linear transformation of the standard $z$-score to obtain a mean of 50 and a S.D. of 10). The combined score was computed by standardizing single trial performances in accuracy and reaction time to $T$-scores (i.e., extreme values in the untransformed data corresponded to extreme values in the T-metric) and averaging them within-persons.

In the domain of postural control, we operationalized postural control as the area spanned by COP displacements. Area measures carry the advantage of being less affected by differences between different stance positions than postural control measures that only focus on COP displacements in either the medio-lateral or the anterior-posterior direction [6]. The main dependent variables were $\log$ transformed COP area and raw COP area. In contrast to the commonly used $95 \%$ confidence elliptic area, the COP area measure used in the present analyses was estimated by using the maximum $x-y$ coordinates in $360^{\circ}$ around the arithmetic mean. This computational is more robust against topological irregularities of the COP areas than $95 \%$ confidence ellipses. Furthermore, the area used in the study is a measure of absolute rather than average COP displacements. Thus, it provides an accurate and complete estimate of the area spanned by the COP excursions [47]. It has been shown empirically that standardized COP-displacement measures such as the standard deviations of displacements are less sensitive to experimental manipulations than the actual range of motion [48]. However, this estimation technique is also more vulnerable to single rapid deviations during the trial period. A number of postural control studies transformed COP-displacement data logarithmically or used a square root transformation to ensure a normal distribution $[1,8,10,29]$. Here, in addition to analyses based on raw scores, we also conducted all analyses with log transformed area measures to attenuate the influence of extreme area values. Furthermore, for comparison purposes we also analyzed the data with the commonly used $95 \%$ confidence elliptic area, as well as the root-meansquare (RMS) of medial-lateral sways. Prior to computing the COP area, the 68-s. COP series were filtered with a sixth-order Butterworth low-pass filter at $6 \mathrm{~Hz}$.

\subsection{Statistical analyses}

The data of this study were analyzed using a mixed design repeated measures ANOVAs. Partialed eta square values $\left(\eta_{\mathrm{p}}^{2}\right)$ are reported as measures of effect size. The effects of cognitive demand and dual-task cost in the cognitive domain and the effect of cognitive demand in postural control were set up as repeated-measures, within-subjects factors. Age group was a between-subjects factor.

We expected that the effect of the level of cognitive demand (varying monotonically from no demand to high demand) would lead to a quadratic function of postural control across conditions. The specificity of the hypothesis allowed a direct test of the theoretically anticipated U-shape function relating cognitive demand and postural control by using polynomial contrasts. In both age groups, we expected a quadratic trend of cognitive demand. In addition, we also expected an age interaction for this trend. We hypothesized that the quadratic trend of older adults would rise at lower levels of cognitive demand than the trend of young adults would. Differential age effects in the decreasing and rising parts of the hypothetical U-function were further validated with a series of planned contrasts. This particular method of analysis was chosen because the use of contrasts is superior to non-directed omnibus tests in terms of specificity and statistical power [54].

\section{Results}

\subsection{Cognitive domain}

A repeated measures ANOVA was conducted to test the between-subjects effect of age (young versus old) and the withinsubjects effects of cognitive demand (choice reaction versus verbal 2-back), and dual-tasking (single-task versus dual-task) on cognitive performance. ${ }^{1}$ Results are presented in Fig. 1. With regard to accuracy, there was a significant effect of age, $F(1$, $37)=30.95, p<0.05, \eta_{\mathrm{p}}^{2}=0.46$, a significant effect of cognitive

\footnotetext{
${ }^{1}$ To rule out possible influences of learning or fatigue on the dualtask effects, we ran the same repeated measurement ANOVAs with trial number as an additional repeated measurement factor. Although significant learning trends across trials were obtained in all indicators of cognitive performance, these trends did not interact with the dual-tasking manipulation or any age by dual-task interaction that we are specifically interested in (accuracy: trial $\times$ dual, $p>0.40$; trial $\times$ dual $\times$ age, $p>0.35$; trial $\times$ dual $\times$ task, $p>0.40$; trial $\times$ dual $\times$ age $\times$ task, $p>0.65$; reaction time: trial $\times$ dual, $p>0.65$; trial $\times$ dual $\times$ age, $p>0.30$; trial $\times$ dual $\times$ task, $p>0.85$; trial $\times$ dual $\times$ age $\times$ task, $p>0.45 ; T$-score: trial $\times$ dual, $p>0.20$; trial $\times$ dual $\times$ age, $p>0.20$; trial $\times$ dual $\times$ task, $p>0.45$; trial $\times$ dual $\times$ age $\times$ task, $p>0.30$ ).
} 

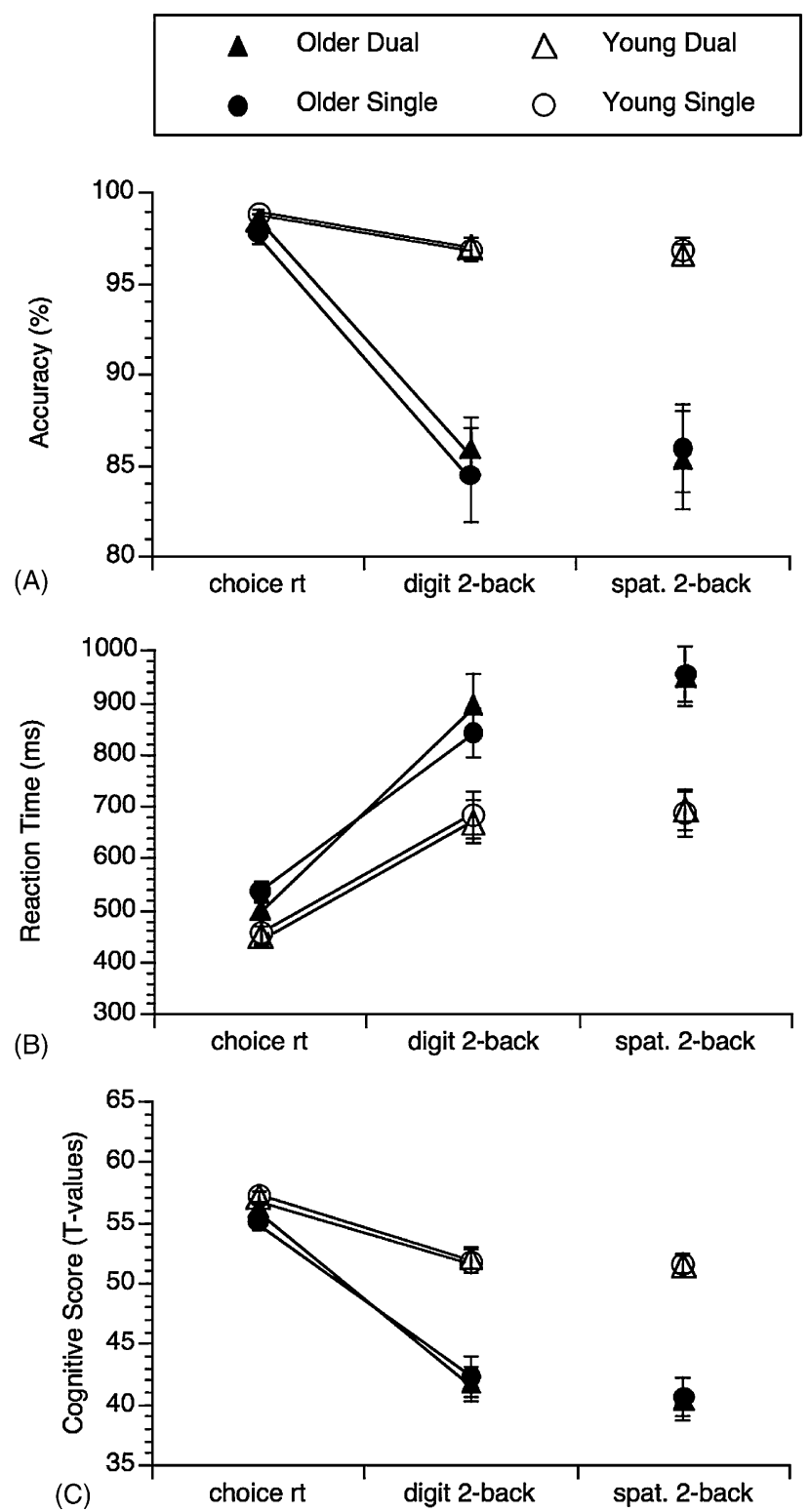

Fig. 1. Cognitive performances displayed as a function of task, age group, and dual-task manipulation. (A) Performances quantified by percentage correct (\%). (B) Performances expressed with respect to response times (ms). (C) Performances indicated by a joint score ( $T$-values). Note: spat, spatial; error bars represent standard errors.

demand, $F(1,37)=71.59, p<0.05, \eta_{\mathrm{p}}^{2}=0.66$, and a significant age by cognitive demand interaction, $F(1,37)=41.97, p<0.05$, $\eta_{\mathrm{p}}^{2}=0.53$. All other effects of dual-tasking on accuracy were not statistically significant. With respect to reaction time, significant effects were observed for age, $F(1,37)=10.80, p<0.05, \eta_{\mathrm{p}}^{2}=$ 0.23 , cognitive demand, $F(1,37)=94.98, p<0.05, \eta_{\mathrm{p}}^{2}=0.72$, and age by cognitive demand, $F(1,37)=4.77, p<0.05, \eta_{\mathrm{p}}^{2}=$ 0.11 . The main effect of dual-tasking on reaction time was not significant. The interaction of cognitive demand $\times$ dual-tasking, however, was marginally significant, $F(1,37)=3.55, p=0.07$, $\eta_{\mathrm{p}}^{2}=0.09$, and a significant age $\times$ cognitive demand $\times$ dualtasking interaction, $F(1,37)=4.29, p<0.05, \eta_{\mathrm{p}}^{2}=0.10$, was observed as well.
To summarize results for accuracy and reaction time: Accuracy levels were lower (Fig. 1A) and reaction times longer (Fig. 1B) for the more demanding digit 2-back working memory task than for the simple choice-reaction time (choice RT) task. Older adults made more errors and were slower than young adults. In line with the age-by-complexity interaction found in numerous cognitive aging experiments (e.g., [58]), age differences were more pronounced with the more demanding task. In the choice-reaction time task, older adults showed faster responses in the sitting condition than in the standing condition. In the digit 2-back task, the opposite pattern was found. At the same time, older adults showed a non-significant trend to be more accurate under dual-task conditions in comparison to single-task conditions, pointing to a speed-accuracy trade-off.

To address the speed-accuracy trade-off, a joint performance score combining accuracy level and reaction time was computed after transforming both performance measures into Tmetric (see above). Fig. 1C summarizes the effect of age, cognitive demand, and dual-tasking on overall cognitive performance. Significant main effects of age, $F(1,37)=33.87$, $p<0.05, \eta_{\mathrm{p}}^{2}=0.49$, and cognitive demand, $F(1,37)=150.58$, $p<0.05, \eta_{\mathrm{p}}^{2}=0.80$, and a significant age $\times$ cognitive demand interaction, $F(1,37)=29.44, p<0.05, \eta_{\mathrm{p}}^{2}=0.45$, were found. Neither the main effect of single versus dual-task condition nor the interactions of dual-tasking with age or cognitive demand were significant. This pattern suggests that older adults adopted a more conservative speed-accuracy criterion in their cognitive performance in dual-task conditions than in single-task conditions.

The digit (i.e., verbal) and spatial versions of the 2-back working memory task were examined for differences in difficulty by conducting an age (young versus old) by modality (verbal versus spatial) by dual-tasking (single-task versus dual-task) repeated measurement ANOVA separately for accuracy and reaction times. For accuracy, only a significant main effect of age was found, $F(1,36)=33.43, p<0.05, \eta_{\mathrm{p}}^{2}=0.48$. Young adults were more accurate than older adults. For reaction times, again, a significant main effect of age was found, $F(1,36)=15.28, p<0.05$, $\eta_{\mathrm{p}}^{2}=0.30$. Participants performed the spatial version slower than the verbal version, $F(1,36)=6.30, p<0.05, \eta_{\mathrm{p}}^{2}=0.15$. In addition, we noted a marginally significant age $\times$ modality interaction, $F(1,36)=3.21, p=0.08, \eta_{\mathrm{p}}^{2}=0.08$, suggesting that older adults showed a more pronounced difference between verbal and spatial versions of the working memory task than young adults. The main effect of dual-tasking and all interactions with the other factors were not significant. For the combined score, only the main effect of age, $F(1,36)=45.20, p<0.05, \eta_{\mathrm{p}}^{2}=0.56$, was significant, again suggesting that performance differences between the two tasks were due to age differences in speedaccuracy trade-offs.

\subsection{Postural control domain}

A repeated measures ANOVA was conducted to test the effects of the between-subject factor of age group (young 

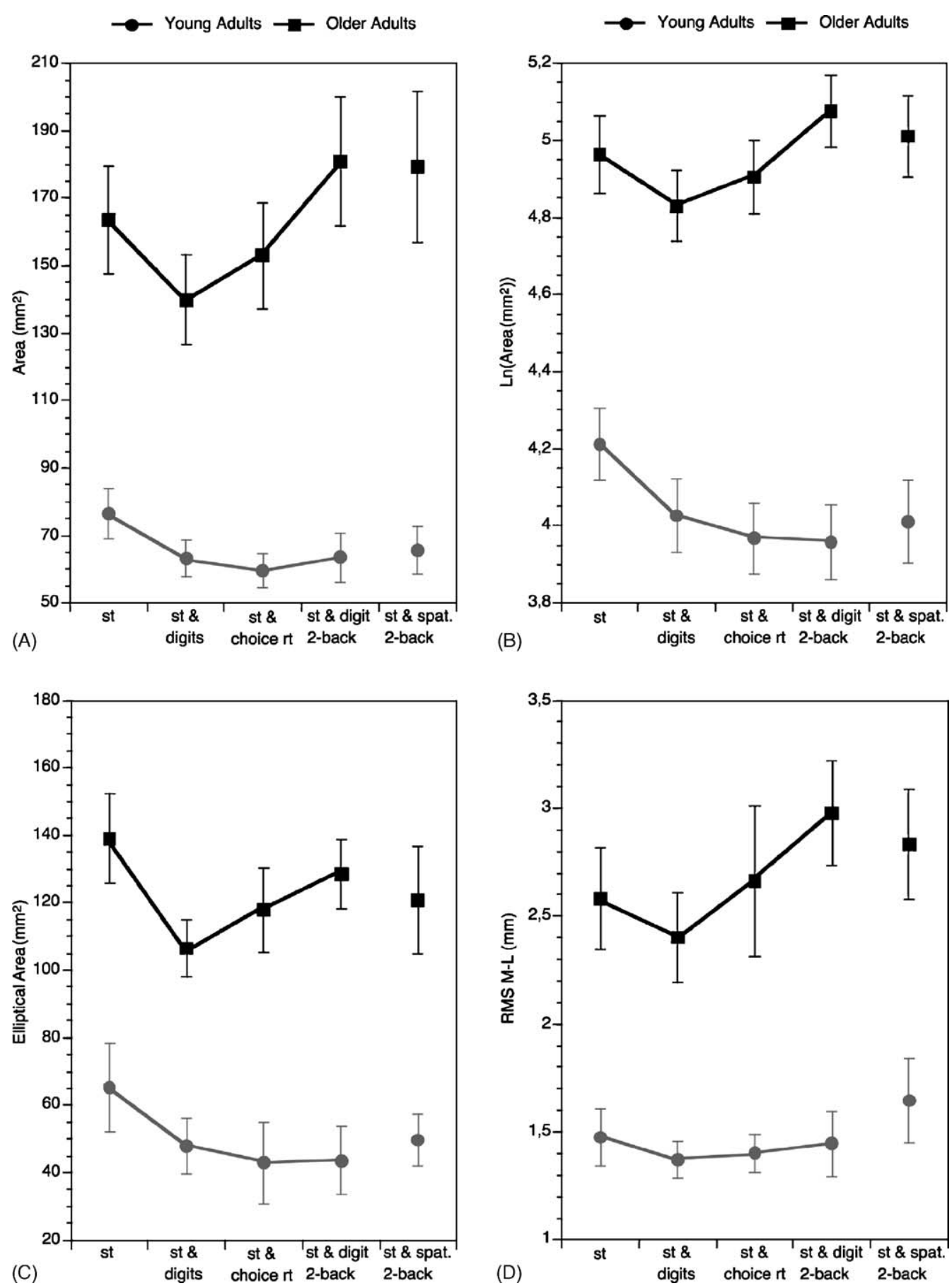

Fig. 2. Postural control performances displayed as a function of task and age group. (A) Postural control indexed with COP area ( $\mathrm{mm}^{2}$ ). (B) Postural control indexed with the natural logarithm of COP area $\left(\ln \left[\mathrm{mm}^{2}\right]\right)$. (C) Postural control indexed with the elliptical COP area sway area (mm²). (D) Postural control indexed with the RMS in medio-lateral direction (mm). Note: The positions of the $x$-axes do not correspond to zero; spat, spatial; st, standing condition; error bars represent standard errors.

versus old), and the two within-subject factors cognitive demand (choice reaction versus digit 2-back), and dual-tasking (single-task versus dual-task) on postural sway. ${ }^{2}$ As shown in Fig. 2, we found a main effect of cognitive demand,

\footnotetext{
${ }^{2}$ We controlled for potential influences of fatigue and learning effects on age by task interactions by running the same repeated measurement ANOVAs with trial number as an additional repeated measurement factor. Neither the main effect of trial number nor any interaction with task or with age by task interactions
}

$F(3,35)=11.41, p<0.05, \eta_{\mathrm{p}}^{2}=0.49$ (raw COP area), $F(3$, $35)=7.77, p<0.05, \eta_{\mathrm{p}}^{2}=0.40$ (log-transformed COP area). The main effect of age on postural control was also significant, $F(1,37)=44.33, p<0.05, \eta_{\mathrm{p}}^{2}=0.55$ (raw COP area), $F(1$,

was found (COP area: trial, $p>0.35$; trial $\times$ task, $p>0.35$; trial $\times$ task $\times$ age, $p>0.40$; ln COP area: trial, $p>0.25$; trial $\times$ task, $p>0.75$; trial $\times$ task $\times$ age, $p>0.45$ ). 
$37)=56.97, p<0.05, \eta_{\mathrm{p}}^{2}=0.61$ (log-transformed COP area). Moreover, the age $\times$ cognitive demand interaction was significant for the log-transformed COP area, $F(3,35)=2.96, p<0.05$, $\eta_{\mathrm{p}}^{2}=0.20$, and marginally significant for the raw COP area, $F(3$, $35)=2.39, p=0.09, \eta_{\mathrm{p}}^{2}=0.17$.

Polynomial contrasts were specified to examine the shape of the interaction between external focus and cognitive demand more directly. The main effect of cognitive demand was associated with a significant quadratic trend, $F(1,37)=20.99$, $p<0.05, \eta_{\mathrm{p}}^{2}=0.36$ (raw COP area), $F(1,37)=20.79, p<0.05$, $\eta_{\mathrm{p}}^{2}=0.36$ (log-transformed COP area). For raw COP area, a quadratic age $\times$ cognitive demand interaction was found, $F(1$, $37)=5.15, p<0.05, \eta_{\mathrm{p}}^{2}=0.12$, whereas for log-transformed $\mathrm{COP}$ area, the age $\times$ cognitive demand interaction was linear, $F(1,37)=8.35, p<0.05, \eta_{\mathrm{p}}^{2}=0.18$. These results show that the function relating cognitive demand to postural control differed by age group. This interaction manifests itself either in a linear shift of the apex or an increase in curvature of the Ushaped function for the older adults in contrast to the young adults, depending on whether raw or log-transformed COP areas are used. Both findings are in line with the prediction that the postural control system of young adults is less susceptible to the effects of cognitive demand than that of older adults.

A series of planned follow-up contrasts was conducted for each age group separately. The standing and digits condition was associated with lower COP displacements than the standing only condition both in young adults, $F(1,19)=8.08$, $p<0.05, \eta_{\mathrm{p}}^{2}=0.30$ (raw COP area), $F(1,19)=8.81, p<0.01$, $\eta_{\mathrm{p}}^{2}=0.32$ (log-transformed COP area), and in older adults, $F(1$, $18)=9.79, p<0.05, \eta_{\mathrm{p}}^{2}=0.35$ (raw COP area), $F(1,18)=6.19$, $p<0.05, \eta_{\mathrm{p}}^{2}=0.26$ (log-transformed COP area). Contrasting the standing and digit choice RT condition with the cognitively more demanding standing and digit 2-back condition demonstrated that young adults did not show a significant difference, $F(1,19)=0.36, p>0.5$ (raw COP area), $F(1,19)=0.03$, $p>0.5, \eta_{\mathrm{p}}^{2}=0.00$ (log-transformed COP area). In contrast, for the same comparison, older adults showed a significant difference in log-transformed COP area, $F(1,18)=4.78, p<0.05$, $\eta_{\mathrm{p}}^{2}=0.21$, and a marginal difference in raw COP area, $F(1$, 19) $=3.16, p=0.09, \eta_{\mathrm{p}}^{2}=0.15$, indicating higher postural sway in the standing and digit 2-back condition than in the standing and digit choice RT condition. As shown in Fig. 2 (comparing panels $\mathrm{A}$ and $\mathrm{B}$ panels $\mathrm{C}$ and $\mathrm{D}$ ), the overall patterns of results are similar across all four types of measures, albeit the age $\times$ task quadratic contrasts are only marginally significant for the elliptic area, $F(1,37)=2.96, p=0.09, \eta_{\mathrm{p}}^{2}=0.07$ and not significant for the medio-lateral RMS $F(1,37)=1.08, p>0.10$, $\eta_{\mathrm{p}}^{2}=0.03$.

An age (young versus old) by cognitive modality (digit 2back versus spatial 2-back) repeated measurement ANOVA was conducted to examine whether postural control is more strongly influenced by spatial processing than by verbal processing. Only the main effect of age, $F(1,37)=36.48, p<0.05, \eta_{\mathrm{p}}^{2}=0.50$ (raw COP area), $F(1,37)=62.91, p<0.05, \eta_{\mathrm{p}}^{2}=0.63(\log -$ transformed $\mathrm{COP}$ area), was significant.

\section{Discussion}

Through monotonic variations of cognitive-demand levels, this study tested the theoretical proposition that postural control sway and cognitive demand are related to each other by a Ushaped function. We also proposed that this relationship would be modulated by individual differences in attentional capacity. We examined the role of attentional capacities by contrasting young and old adults, given evidence of clear negative adult age differences in cognitive efficacy.

\subsection{Summary of results}

In single and dual-task conditions, older adults showed lower postural control performance and lower cognitive performance than young adults. With the joint accuracy and reaction time scores, no dual-task effect was observed in the cognitive domain, indicating that performing these cognitive tasks while sitting and standing yielded the same results. The effect of dual-tasking on cognition was expressed in terms of a more conservative speed-accuracy trade-off for cognitive performance, but not in performance decrements as such.

The absence of dual-task costs in the cognitive domain has the fortunate consequence that it renders dual-task benefits and costs in postural control domain more readily interpretable, including the modulation of these differences by age. Regarding dualtask effects in postural control, the effect of cognitive demand showed a clear quadratic trend in both age groups. In young adults, this trend was due to a reduction in the area of COP displacements found in all dual-task experimental conditions. In older adults, the effect of cognitive demand on postural sway followed the predicted U-shaped function. Compared to a situation in which standing still was the only task to perform, adding a simple perceptual task (standing and viewing digits) reduced COP displacements in both young and old adults. This reduction effect cannot be attributed to the beneficial effect of visual fixation alone because participants were required to focus on a fixation cross in the standing only condition as well. In older adults, however, the beneficial effect of a secondary cognitive task on postural control performance diminished as the cognitive demand of the secondary task increased. Although the general shape of this interaction was identical for different measures of COP displacements it reached statistical significance only in measures that considered the actual range of motion. This pattern of results replicates former findings [48] and might implicate that attentional resource competition in dual-tasking postural control also results in short-term lapses in postural control behavior. Additional analyses also showed that postural control was not more strongly affected by spatial processing than by verbal processing when task difficulty was taken into account.

\subsection{Implications for related studies and future research}

Our results provide little support for a theoretical position that frames the interaction between postural control and cognition entirely in terms of attentional resource competition. Rather, this study supports the proposition of a U-shaped relation 
between cognitive processing demands and postural control $[11,51,68,69]$ by providing direct empirical support on the basis of an experiment with monotonically increased levels of cognitive demands and individual differences in attentional capacity. Furthermore, the contrast between young and old adults confirms that individual differences in attentional capacity modulate the point at which a given level of cognitive demands change from being beneficial or detrimental for postural control.

Several authors have argued that the U-shaped relation between cognitive demand and postural sway might reflect the level of arousal associated with the secondary cognitive demand according to the Yerkes-Dodson law $[11,51,69]$. According to this interpretation, low cognitive demands increase the level of arousal in a way that triggers optimal postural sway regulation, whereas high cognitive load leads to higher levels of arousal that are suboptimal, causing deterioration of performance. Studies contrasting directly indicators of arousal, such as skin conductance and heart rate, in single-task and dual-task situations, however, have provided mixed results, with increments [4,10,29], decrements [10], or no changes [29] in arousal as a function of cognitive demand. Furthermore, a correlational analysis aimed at linking the level of arousal to the amount of COP displacements across a trial-length of $75 \mathrm{~s}$ did not reveal any significant relationship between the two [29]. Considering these results together, we refrain from an arousal-based explanation for the U-shaped dual-task interaction, although our data cannot directly rule out this possibility.

In our view, the results of the present study suggest a dualprocess account of the relation between postural sway and cognition. The first process is associated with the decreasing part of the U-shaped interaction, or put differently, the beneficial range of the secondary task. Given low cognitive demands, secondary cognitive activities improve postural performance by shifting the focus of overt attention away from a highly automatized activity. In line with previous findings $[35,53,78]$, we found that a concurrent perceptual task of low cognitive demand decreased postural sway in both age groups in contrast to a simple standing condition. In the stand-only condition, participants adopted an internal attentional focus trying to control their postural sway in a conscious manner. According to the hypothesis of constrained action [77], focusing attention on a highly automatized behavior such as postural control interferes, rather than helps, the automatic control processes. In contrast, an external focus of attention, which is provided by the secondary perceptual task, enables the postural control system to self-organize automatically. Therefore, postural sway decreased in our experiment when a perceptual task of low cognitive demand was added.

The second process determines primarily the rising part of the U-shaped interaction. Given high demands of a secondary cognitive task, cognitive activities hinder postural control through cross-domain resource competition. In older adults, the beneficial effect of the secondary task in providing an external focus of attention was overtaken by the negative effect of resource competition as the cognitive demand of the secondary task increased. Contrasting the results of young and older adults underscores that the effects of cognitive demand on postural control should be considered in relative rather than absolute terms. A sec- ondary task with a given level of cognitive demand may have different functional effects on individuals that differ in their amount of attentional resources. Due to older adults' more limited attentional capacity, the cognitively more demanding 2-back working memory tasks resulted in attentional resource competition between cognition and postural control performance, and consequently affected postural control negatively. In contrast, due to their relatively higher level of cognitive capacity, young adults did not experience increasing resource competition with the same nominal level of cognitive demands and their postural performance was not impaired under the dual-tasking conditions.

As for the notion that variations in stimulus and response modalities of the secondary cognitive tasks might play a major role in affecting in COP displacements in old adults under dualtask conditions, our results provide little support for it. For instance, in older adults, postural sway increased significantly from simple choice response to the more demanding 2-back working memory tasks although stimuli and response modalities were identical for the two tasks (i.e., 22 button presses per trial). Furthermore, in contrast to earlier results [17,32], our results do not suggest that the interference between postural control and cognition is modality specific. Spatial processing did not influence postural control to a greater degree than verbal processing. Rather, our results support the hypothesis that cognitive tasks with similar levels of attentional demands also have similar effects on sensorimotor processes (cf. [8,25,78]). Future studies with sufficient statistical power should examine in more detail to what extent stimulus and processing modalities nested within demand levels influence the direction and strength of effects on postural performance. In our view, the difficulty (i.e., demand level) of the cognitive task plays a fundamental role in shaping the dual-task relation between cognition and postural control. At the same time, the focus of attention and individual differences in attentional capacity need to be considered as well.

Mitra and Frazier [40] contrasted dual-task performances across conditions in which participants were either instructed to give equal emphasis to postural control and cognitive performance or to focus exclusively on the cognitive task. They found that even with increasing cognitive load less postural sway was observable in the equal-emphasis condition than in the focus on cognition condition. At first glance, these results seem to contradict the general notion that it is maladaptive to allocate attention on highly automatized postural control process. However, the specific effect of instruction reported by Mitra and Frazier [40] might be limited to a dual-task situation in which postural control and cognition compete for limited attentional resources (i.e., the rising part of the U-shaped interaction). Unfortunately, the present study does not address the issue of deliberate allocation of attentional resources to either one of the two domains of functions. The suggested dual-process model would predict, however, that the effect of instruction would depend on the individual's position on the U-shaped function. In the beneficial range of the secondary task by postural control interactions, a focus on the sensorimotor performance should increase COP displacements. In the range of attentional resource competition, 
however, a focus on postural control would decrease postural sway by simultaneously sacrificing performance on the cognitive task (e.g., [40]).

A natural extension from our results is to experimentally manipulate the sensorimotor demand in dual-tasking postural control in order to gain a more complete understanding of the complex postural control and cognition interaction. Including postural constraints into future study designs may clarify why the young adults in our sample did not show an increase in postural sway with increasing cognitive demands. One possible reason is that the levels of cognitive demands used in our study were not difficult enough for young adults to show effects of attentional competition when performing a rather natural, easy postural control task, i.e., simple standing. A number of studies have shown that increasing the difficulty of the postural task does increase the probability of attentional resource competition (e.g., $[9,19,50,68,79])$. Future studies should, therefore, examine the postural control system of healthy young adults under conditions of higher levels of dual-task demands to explore its limits. Perhaps, simple standing is so highly automatized in young adults that increasing the cognitive load might be insufficient to tax their postural control system. Therefore, other experimental paradigms such as walking on a narrow track $[23,25]$ may be needed to increase the demand characteristics of the postural task to observe dual-task in postural control among young adults. Note that significant dual-task costs in cognitive performance have been found even in young gymnastics experts when they were standing on one leg [68].

Moreover, the perceived sensorimotor challenge might determine to some degree whether attentional resources are invested into postural control or into cognitive performance during dualtasking. Patterns of resource allocation may also differ by age. The "posture-first hypothesis" would predict that older adults focus more strongly on their postural control than on their cognitive performance to avoid falls [59], at least under conditions of postural threat. A number of studies have examined the interactions between cognitive demands and postural control under postural constraints (e.g., $[9,36,50,59,63])$. Two of these studies found an increase in postural control in dual-task situations in older adults but not in young adults under challenging postural control demands $[36,63]$. The interpretation of these results is complicated by lack of information about age differences in dual-task cognitive performance or speed-accuracy trade-offs in cognitive performance. A third study [4], however, manipulated directly the level of threat without altering the sensorimotor demands by contrasting different surface heights on which participants stood and assessing both the speed and accuracy of the cognitive performance. Under highly threatening conditions, older adults reduced their postural sway when a cognitive task was added whereas young adults did not display a significant difference. More importantly, the increased postural control among the older adults was associated with lower cognitive performance, in line with the limited attentional capacity view (see also [47]). Note again that a nominally identical level of postural challenge may translate into the same degree of postural change relative to baseline levels in young and older adults. Clearly, future research aiming at manipulating both cognitive demands and postural constraints needs to consider appropriate levels of age-comparative manipulations.

\section{Conclusion}

The results of the present study suggest a dual-process account of the relation between postural sway and cognition. Firstly, cognitive activities improve postural performance by shifting the focus of attention away from a highly automatized activity. Beneficial effects are more easily observed at low levels of cognitive task difficulty because such levels are sufficient to shift attention away from the postural domain without causing resource competition. Secondly, cognitive activities hinder postural control through cross-domain resource competition. Thus, detrimental effects are found only at higher levels of cognitive task difficulty, when resource competition actually sets in. Accordingly, the transition from beneficial to detrimental effects occurs at lower levels of nominal task difficulty for individuals with lower task-relevant resources such as older adults than for individuals with higher task-relevant resources such as young adults. Future studies need to study and identify the nature and interactions among mechanisms that mediate effects of attention on bottom-up and top-down components of postural regulation.

\section{Acknowledgements}

The work reported in this article was conducted as part of the Intra-Person Dynamics Project at the Max Planck Institute for Human Development, Center for Lifespan Psychology. We thank Paul B., Baltes, Ralf Krampe, Martin Lövdén, Christina Röcke, and Jacqui Smith for helpful discussions.

\section{References}

[1] G. Anderssson, J. Hagman, R. Talianzadeh, A. Svedberg, H.C. Larsen, Effect of cognitive load on postural control, Brain Res. Bull. 58 (2002) $135-139$.

[2] G. Andersson, L. Yardley, L. Luxon, A dual-task study of interference between mental activity and control of balance, Am. J. Otol. 19 (1998) 632-637.

[3] P.B. Baltes, U. Lindenberger, Emergence of a powerful connection between sensory and cognitive functions across the lifespan: a new window to the study of cognitive aging? Psychol. Aging 12 (1997) 12-21.

[4] L.A. Brown, R.J. Sleik, M.A. Polych, W.H. Gage, Is the prioritization of postural control altered in conditions of postural threat in younger and older adults? J. Gerontol. Med. Sci. 57A (2002) 785-792.

[5] M.G. Carpenter, J.S. Frank, D.A. Winter, G.W. Peysar, Sampling duration effects of centre of pressure summary measures, Gait Posture 13 (2001) 35-40.

[6] L. Chiari, L. Rocchi, A. Cappello, Stabilometric parameters are affected by anthropometry and foot placement, Clin. Biomech. 17 (2002) 666-677.

[7] J.E. Condron, K.D. Hill, Reliability and validity of a dual-task force platform assessment of balance performance: effect of age, balance impairment, and cognitive task, JAGS 50 (2002) 157-162.

[8] M.C. Dault, J.S. Frank, F. Allard, Influence of a visuospatial, verbal and central executive working memory task on postural control, Gait Posture 14 (2001) 110-116.

[9] M.C. Dault, A.C. Geurts, T.W. Mulder, J. Duysens, Postural control and cognitive task performance in healthy participants while balancing 
on different support-surface configurations, Gait Posture 14 (3) (2001) $248-255$.

[10] M.C. Dault, L. Yardley, J.S. Frank, Does articulation contribute to modifications of postural control during dual-task paradigms? Cogn. Brain Res. (2003) 434-440.

[11] D. Deviterne, G.C. Gauchard, M. Jamet, G. Vancon, P.P. Perrin, Added cognitive load through rotary auditory stimulation can improve the quality of postural control in the elderly, Brain Res. Bull. 64 (6) (2005) 487-492.

[12] T. Ehrenfried, M. Guerraz, K.V. Thilo, L. Yardley, M.A. Gresty, Posture and mental task performance when viewing a moving visual field, Cogn. Brain Res. 17 (2003) 140-153.

[13] M. Inoue, T. Suhara, Y. Sudo, Y. Okubo, F. Yasuno, T. Kishimoto, K. Yoshikawa, S. Tanada, Age-related reduction of extrastriatal dopamine D2 receptor measured by PET, Life Sci. 69 (2001) 1079-1084.

[14] M. Jamet, D. Deviterne, G.C. Gauchard, G. Vancon, P.P. Perrin, Higher visual dependency increases balance control perturbation during cognitive task fulfillment in elderly people, Neurosci. Lett. 359 (2004) 61-64.

[15] M.K. Johnson, K.J. Mitchel, C.L. Raye, E.J. Greene, An age-related deficit in prefrontal cortical function associated with refreshing information, Psychol. Sci. 15 (2004) 127-132.

[16] V. Kaasinen, H. Vilkman, J. Hietala, K. Nagren, H. Helenius, H. Olsson, L. Farde, J.O. Rinne, Age-related dopamine D2/D3 receptor loss in extrastrial regions of the human brain, Neurobiol. Aging 21 (2000) 683-688.

[17] B. Kerr, S.M. Condon, L.A. McDonald, Cognitive spatial processing and the regulation of posture, J. Exp. Psychol. Hum. 11 (1985) 617-622.

[18] E. Labyt, W. Szurhaj, J.-L. Bourriez, F. Cassim, L. Defebvre, A. Destee, J.-D. Guieu, Ph. Derambure, Changes in oscillatory cortical activity related to a visoumotor task in young and elderly healthy subjects, Clin. Neurophys. 114 (2003) 1153-1166.

[19] Y. Lajoie, N. Teasdale, C. Bard, M. Fleury, Attentional demands for static and dynamic equilibrium, Exp. Brain Res. 97 (1993) 139-144.

[20] S.-C. Li, S.H. Aggen, J.R. Nesselroade, P.B. Baltes, Short-term fluctuations in elderly people's sensorimotor function predict text and spatial memory performance: the MacArthur successful aging studies, Gerontology 47 (2001) 100-116.

[21] S.-C. Li, H.R. Dinse, Aging of the brain, sensorimotor, and cognitive processes, Neurosci. Biobehav. Rev. 26 (2002) (edited special issue).

[22] K.Z.H. Li, U. Lindenberger, Relations between aging sensory/sensorimotor and cognitive functions, Neurosci. Biobehav. Rev. 26 (2002) 777-783.

[23] K.Z.H. Li, U. Lindenberger, A.M. Freund, P.B. Baltes, Walking while memorizing: age-related differences in compensatory behavior, Psychol. Sci. 12 (2001) 230-237.

[24] U. Lindenberger, P.B. Baltes, Sensory functioning and intelligence in old age: a strong connection, Psychol. Aging 9 (1994) 339355.

[25] U. Lindenberger, M. Marsiske, P.B. Baltes, Memorizing while walking: increase in dual-task costs from young adulthood to old age, Psychol. Aging 15 (2000) 417-436.

[26] L.A. Lipsitz, Dynamics of stability: the physiological basis of functional health and frailty, J. Gerontol. Biol. Sci. 57A (2002) B115-B125.

[27] B.J.W. Losier, R.M. Klein, A review of the evidence for a disengage deficit following parietal lobe damage, Neurosci. Biobehav. Rev. 25 (2001) $1-13$.

[28] L. Lundin-Olsson, L. Nyberg, Y. Gustafson, Stop walking when talking as a predictor of falls in elderly people, Lancet 349 (1997) 617.

[29] B.E. Maki, W.E. McIlroy, Influence of arousal and attention on the control of postural sway, J. Vestib. Res. 6 (1996) 53-59.

[30] B.E. Maki, A. Zecevic, H. Bateni, N. Kishenbaum, W.E. McIllroy, Cognitive demands of executing postural reactions: does aging impede attention switching? Neuroreport 12 (2001) 3583-3587.

[31] J. Massion, Postural control system, Curr. Opin. Neurobiol. 4 (1994) 877-887.

[32] E.A. Maylor, A.M. Wing, Age differences in postural stability are increased by additional cognitive demands, J. Gerontol. Psychol. Sci. 51B (2000) P143-P154.
[33] W.E. McIlroy, B.E. Maki, Preferred placement of the feet during quiet standing: development of a standardized foot placement for balance testing, Clin. Biomech. 12 (1997) 66-70.

[34] N.H. McNevin, C.H. Shea, G. Wulf, Increasing the distance of an external focus of attention enhances learning, Psychol. Res. 67 (2003) 22-29.

[35] N.H. McNevin, G. Wulf, Attentional focus on supra-postural affects postural control, Hum. Mov. Sci. 21 (2002) 187-202.

[36] I. Melzner, N. Benjuya, J. Kaplanski, Age-related changes of postural control: effect of cognitive tasks, Gerontology 47 (2001) 189-194.

[37] T. Mergner, T. Rosemeier, Interaction of vestibular, somatosensory and visual signals for postural control under terrestrial and microgravity conditions-a conceptual model, Brain Res. Rev. (1998) 118-135.

[38] C. Michel, Y. Rosseti, G. Rode, C. Tikikete, After-effects of visuomanual adaptation to prisms on body posture in normal subjects, Exp. Brain Res. 148 (2003) 219-226.

[39] S. Mitra, Postural costs of suprapostural task load, Hum. Mov. Sci. 22 (2003) 253-270.

[40] S. Mitra, E.V. Fraizer, Effects of explicit sway-minimization on postural-suprapostural dual-task performance, Hum. Mov. Sci. 23 (2004) $1-20$.

[41] A. Mouzat, M. Dabonneville, P. Bertrand, The effect of feet position on orthostatic posture in a female sample group, Neurosci. Lett. 365 (2004) $79-82$.

[42] H. Pashler, Attentional limitations in doing two things at the same time, Curr. Dir. Psychol. Sci. 1 (1992) 44-48.

[43] G.L. Pellecchia, Postural sway increases with attentional demands of concurrent cognitive task, Gait Posture 18 (2003) 29-34.

[44] D.A. Pérennou, C. Leblond, N. Amblard, J.P. Micallef, C. Hérisson, J.Y. Pélissier, Transcutaneous electric nerve stimulation reduces neglectrelated postural instability after stroke, Arch. Phys. Med. Rehabil. 82 (2001) 440-447.

[45] M.I. Posner, Orienting of attention, Q. J. Exp. Psychol. 32 (1980) 3-25.

[46] M.I. Posner, J.A. Walker, F.J. Friedrich, R.D. Rafal, How do the parietal lobes direct covert attention? Neuropsychologia 25 (1987) 135-145.

[47] M.A. Rapp, R.T. Krampe, P.B. Baltes, Adaptive task prioritization in aging: selective resource allocation to postural control is preserved in Alzheimer disease, Am. J. Geriatr. Psychiatry 14 (2006) 52-61.

[48] J.A. Raymakers, M.M. Samson, H.J.J. Verhaar, The assessment of body sway and the choice of stability parameters, Gait Posture 21 (2005) $48-58$.

[49] N. Raz, F. Gunning-Dixon, D. Head, K.M. Rodrique, A. Williamson, J.D. Acker, Aging, sexual dimorphism, and hemispheric asymmetry of the cerebral cortex: replicability of regional differences in volumes, Neurobiol. Aging 25 (2004) 377-396.

[50] M.S. Redfern, J.R. Jennings, C. Martin, J.M. Furman, Attention influences sensory integration for postural control in older adults, Gait Posture 14 (2001) 211-216.

[51] M.A. Riley, A.A. Baker, J.M. Schmit, Inverse relation between postural variability and difficulty of a concurrent short-term memory task, Brain Res. Bull. 62 (2003) 191-195.

[52] M.A. Riley, A.A. Baker, J.M. Schmit, E. Weaver, Effects of visual and auditory short-term memory tasks on the spatiotemporal dynamics and variability of postural sway, J. Mot. Behav. 37 (2005) 311-324.

[53] M.A. Riley, T.A. Stoffregen, M.J. Grocki, M.T. Turvey, Postural stabilization for the control of touching, Hum. Mov. Sci. 18 (1999) 553-571.

[54] R. Rosenthal, R.L. Rlosnow, Contrast Analysis, Cambridge University Press, London, 1985

[55] J. Rowe, K. Friston, R. Frackowiak, R. Passingham, Attention to action: specific modulation of corticocortical interactions in humans, NeuroImage 17 (2002) 988-998.

[56] M.F.S. Rushworth, H. Johansen-Berg, S.M. Göbel, J.T. Devlin, The left parietal and premotor cortices: motor attention and selection, NeuroImage 20 (2003) S89-S100.

[57] B. Rypma, V. Prabhakaran, J.E. Desmond, J.D.E. Gabrieli, Age differences in prefrontal cortical activity in working memory, Psychol. Aging 16 (2001) 371-384.

[58] T.A. Salthouse, Why do adult age-differences increase with task complexity? Dev. Psychol. 28 (1992) 905-918. 
[59] A. Shumway-Cook, M. Wollacott, Attentional demands and postural control: the effect of sensory context, J. Gerontol. Biol. Sci. 55A (2000) M10-M16.

[60] A. Shumway-Cook, M. Wollacott, K.A. Kerns, M. Baldwin, The effects of two types of cognitive tasks on postural stability in older adults with and without a history of falls, J. Gerontol. Biol. Sci. 52A (1997) M232-M240.

[61] T.A. Stoffregen, R.J. Pagulayan, B.G. Bardy, L.J. Hettinger, Modulating postural control to facilitate visual performance, Hum. Mov. Sci. 19 (2000) 203-220.

[62] T.A. Stoffregen, L.J. Smart, B.G. Bardy, R.J. Pagulayan, Postural stabilization of looking, J. Exp. Psychol. Hum. 25 (1999) 1641-1658.

[63] L. Swan, H. Otani, P.V. Loubert, S.M. Sheffert, G.L. Dunbar, Improving balance by performing a secondary cognitive task, Br. J. Psychol. 95 (2004) 31-40.

[64] N. Teasdale, M. Simoneau, Attentional demands for postural control: the effects of aging and sensory reintegration, Gait Posture 14 (2001) 203-210.

[65] P. Their, P.O. Karnath (Eds.), Parietal Lobe Contribution to Orientation in 3D Space, Springer-Verlag, Heidelberg, 1997.

[66] C. Tikikete, G. Rode, Y. Rossetti, J. Pichon, L. Li, D. Boisson, Prism adaptation to rightward optical deviation improves postural imbalance in left-hempiparetic patients, Curr. Biol. 11 (2001) 524-528.

[67] D.J. Tisserand, M.P.J. van Boxtel, J.C. Pruessner, P. Hofman, A.C. Evans, J. Joilles, A voxel-based morphometric study to determine individual differences in gray matter density associated with age and cognitive change over time, Cereb. Cortex 14 (2004) 966-1073.

[68] N. Vuillerme, V. Nougier, Attentional demand for postural sway: the effect of expertise in gymnastics, Brain Res. Bull. 63 (2004) 161-165.

[69] N. Vuillerme, V. Nougier, R. Camicioli, Veering in human locomotion: modulatory effect of attention, Neurosci. Lett. 331 (3) (2002) 175-178.
[70] N. Vuillerme, V. Nougier, N. Teasdale, Effects of a reaction time task on postural control in humans, Neurosci. Lett. 291 (2) (2000) 77-80.

[71] M.H. Wollacott, Systems contributing to balance disorders in older adults, J. Gerontol. Med. Sci. 55A (2000) M424-M428.

[72] M.H. Wollacott, J.A. Shumway-Cook, Attention and the control of posture and gait: a review of an emerging area of research, Gait Posture 16 (2002) $1-14$

[73] D.M. Wolpert, Z. Ghahramani, J.R. Flanagan, Perspectives and problems in motor learning, Trends Cogn. Sci. 5 (2001) 487-494.

[74] D.M. Wolpert, Z. Ghahramani, M.I. Jordan, An internal model for sensorimotor integration, Science 269 (1995) 1880-1882.

[75] D.N. Wolpert, S.J. Goodbody, M. Husain, Maintaining internal representations: the role of the human superior parietal lobe, Nat. Neurosci. 1 (1998) 529-533.

[76] D.L. Weeks, R. Forget, L. Mouchino, D. Gravel, D. Bourbonnais, Interaction between attention demanding motor and cognitive tasks and static postural stability, Gerontology 49 (2003) 225-232.

[77] G. Wulf, N. McNevin, C.H. Shea, The automaticity of complex motor skill learning as a function of attentional focus, Q. J. Exp. Psychol. 54 (2001) 1143-1154.

[78] G. Wulf, J. Mercer, N. McNevin, M.A. Guadagnoli, Reciprocal influence of attentional focus on postural control and suprapostural task performance, J. Mot. Behav. 36 (2004) 189-199.

[79] L. Yardley, M. Gardener, A. Bronstein, R. Davies, D. Buckwell, L. Luxon, Interference between postural control and mental task performance in patients with vestibular disorder and healthy controls, J. Neurol. Neurosur. Psychiatry 71 (2001) 48-52.

[80] L. Yardley, M. Gardner, A. Leadbetter, N. Lavie, Effect of articulatory and mental tasks on postural control, Neuroreport 10 (1999) 215219 